\section{Noninvasive measurement of cortical activity during effective brain stimulation for movement disorders}
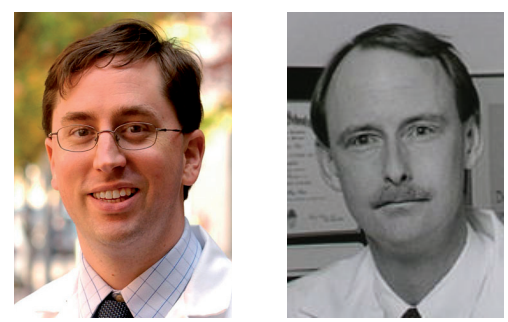

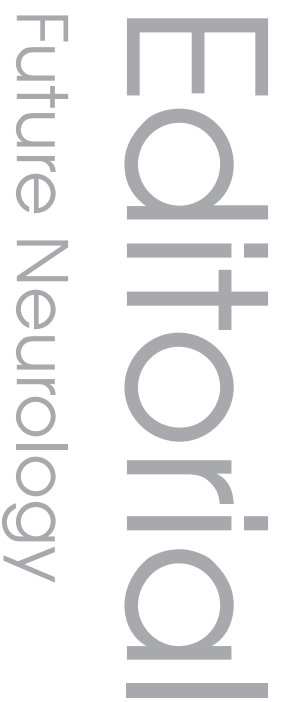

Harrison C Walker*1 \& Barton L Guthrie'

'University of Alabama at Birmingham; 1720 7th Avenue South, Sparks Center 360E, Birmingham. AL 35294-0017, USA

*Author for correspondence: Tel.: +1 2059969540 = Fax: +1 2059964039 n howalker@uab.edu

Deep brain stimulation (DBS) is superior to medical therapy for the motor symptoms of Parkinson's disease, essential tremor and various forms of dystonia. Additionally, DBS is being investigated for use with refractory major depression, obsessive-compulsive disorder, epilepsy and other indications. Despite its use in more than 70,000 patients worldwide, the therapeutic mechanism of DBS is unclear. The aim of this article is to put our recent EEG studies in DBS patients with movement disorders into the larger context of other research in the field. Better understanding of how DBS works can be applied to optimize therapy for patients; to guide innovation, particularly in emerging indications; and to better understand disease pathophysiology.

We have recently demonstrated short latency activation of the cerebral cortex with noninvasive EEG recordings during clinically effective high frequency $(>100 \mathrm{~Hz})$ subthalamic and thalamic DBS for Parkinson's disease and essential tremor [1,2]. Although EEG has excellent temporal resolution, scalp electrodes detect the combined electrical activity from the subcortical electrical stimulus pulse and the cortical potentials of interest. Consequently, prior EEG studies typically evaluated changes in cortical rhythms during symptomatically ineffective low frequency DBS $(2-20 \mathrm{~Hz})$, allowing the observation of event-related potentials (ERPs) between successive stimuli. Even at these low stimulation frequencies, it remains difficult to evaluate the earliest components of the brain response because of the amplitude and width of the stimulus artifact. Consequently, prior EEG studies in humans with DBS only visualize peaks that occur $\geq 3 \mathrm{~ms}$ after stimulus onset [3-6].

Expanding on prior methods, we developed signal analysis techniques to minimize the stimulus artifact, allowing us to distinguish brain activity, both immediately after stimulus onset and during clinically effective high frequency stimulation [3]. Reversing the anode and cathode contacts on the DBS electrode inverts the polarity of the electrical stimulus artifact, but not the underlying brain response. Exploiting this phenomenon, we generated pairs of ERPs across stimulation conditions, summed corresponding pairs to minimize the stimulus artifact and then evaluated changes in cortical activity across a range of clinically effective and ineffective DBS settings. We found that subcortical DBS evokes precisely timed, polyphasic ERPs, with the earliest cortical responses occurring at shorter latencies than had been described in previous studies (approximately $1 \mathrm{~ms}$ after stimulus onset). Importantly, the amplitude of this short latency potential was associated with improvement in clinical symptoms as the DBS voltage was increased.

We have argued that this short latency potential most likely represents retrograde, nonsynaptic (antidromic) activation of the cortex by DBS, based on the following observations: it occurs too rapidly for synaptic activity; it shows precise timing and independence from stimulation frequency; its latency is consistent with the conduction velocities for large, myelinated descending axons of cortical pyramidal cells $(\sim 60 \mathrm{~m} / \mathrm{s})$; it localizes to the ipsilateral somatosensory cortex, not to a deep,

\section{Keywords}

- cerebral cortex = deep brain stimulation $=$ essential tremor w event-related potential

- Parkinson's disease

- subthalamic nucleus

- ventral intermediate nucleus

Future Medicine part of 
midline source (as would be expected for a subcortical potential near the DBS electrode); animal studies have shown antidromic cortical activation with single unit recordings during electrical stimulation of both subcortical targets [7,8]; and transcranial magnetic and paired pulse stimulation paradigms in humans have both provided indirect evidence for this mechanism $[3,6]$. Despite these observations, we were unable to perform single unit collision studies to conclusively verify antidromic activation, because these were noninvasive studies in human subjects. With respect to the later peaks in the ERP ( $\geq 3 \mathrm{~ms}$ latency), potential mechanisms could include synchronized spontaneous cortical bursts following short latency activation, antidromic activation of descending fibers with slower conduction velocities, orthodromic monosynaptic activity and/or polysynaptic activation, mostly likely through the pallidothalamic pathway.

"Speculatively, high-frequency DBS
( $\geq 100 \mathrm{~Hz}$ ) may improve hyperkinetic
involuntary movements such as tremor by
dividing pathological central oscillations
into more rapid, compact temporal units,
such that they are not expressed in the
periphery, based upon motor unit
physiology and/or the biomechanical
properties of the limbs."

There is growing interest in whether the reciprocal connections between cortex and subcortical structures may play a role in movement disorder pathophysiology and the therapeutic mechanism of DBS [9,10]. Gradinaru et al. showed improvement in a mouse model of parkinsonism during selective optical stimulation of frontal cortex neurons and electrical stimulation of the subthalamic region, but not during selective photic stimulation or inhibition of subthalamic neurons alone [11]. Since thalamic and subthalamic DBS both improve tremor from Parkinson's disease and essential tremor, our studies parallel these findings and demonstrate a shared cortical physiology associated with tremor suppression at two different stimulation sites and disease states [12,13].

Despite these data, attempts at epidural motor cortex stimulation (EMCS) in humans with Parkinson disease and essential tremor in small case series have shown mixed results, with a recent blinded study, suggesting that it may improve symptoms of essential tremor, but not Parkinson disease [14]. Although the small sample sizes and potential technical/methodological differences of the various EMCS studies make them difficult to reconcile with one another, they raise the possibility that the cortical physiology associated with subthalamic and thalamic DBS in our recent work might be unrelated to its therapeutic mechanism. While this is possible, these subcortical DBS targets are small, dense functional nodes in the motor network that receive projections from multiple cortical regions, while EMCS activates the cerebral cortex less discriminately over a larger volume. This has lead authors to question whether EMCS influences neuronal activity efficiently enough to exert meaningful behavioral effects [14]. Regardless of whether EMCS sufficiently influences primary motor cortex activity, it still may not act on adjacent cortical regions such as the premotor, supplementary motor and parietal areas (regions with known projections to the subthalamic nucleus and thalamus). Additionally, short latency activation of the cortex during subcortical DBS should only occur in cortical neurons with axonal projections near the stimulation site, whereas EMCS presumably activates multiple populations of cortical principle cells and interneurons less discriminately. Finally, clinical observations of reversible motor seizures, aphasias and other cortical symptoms during EMCS, but not DBS, support the notion that these therapies probably alter cortical physiology in fundamentally different ways.

Despite the comparable symptomatic effects of DBS and focal stereotactic lesions (thalamotomy, pallidotomy and subthalamotomy), functional imaging studies investigating DBS paradoxically show activation both around the active stimulation contact and in adjacent output structures [15]. These findings have called into question ideas about basal ganglia pathophysiology and the mechanism of DBS, both of which were partly outgrowths of the clinical phenomenology of stereotactic lesions. Unlike electrophysiological techniques, the temporal resolution of functional MRI and PET are in the order of seconds [16]. This has particular relevance in movement disorders, which have symptoms that often occur on more rapid time scales. Subthalamic and thalamic DBS, for instance, both improve tremor that oscillates at $4-6 \mathrm{~Hz}$, and DBS improves the velocity of repetitive hand movements and simple reaction times, the latter by only approximately $100 \mathrm{~ms}$ versus no stimulation [17]. Additionally, 
higher stimulation frequencies $(\geq 100 \mathrm{~Hz})$ are generally more effective than lower frequencies across various disease states and stimulation targets, and regular stimulation patterns improve motor symptoms to a greater extent than either random or intermittent DBS delivered at the same total stimulation rate $[18,19]$. Finally, studies at downstream sites from the stimulation target in humans and animal models of disease typically show activation or modulation of neuronal firing patterns during DBS, not inhibition [20,21]. In the context of our recent EEG studies, these collective findings suggest that the precise timing of neuronal activity in the motor system may be more important than the discharge rate at any local structure, at least with respect to the therapeutic mechanism of high-frequency electrical stimulation.

Regardless of the underlying mechanism(s), our findings suggest that cortical neuronal discharges are synchronized or regularized to the stimulation frequency or one of its subharmonics during clinically effective highfrequency stimulation. At least with respect to cortical activity, they do not support the notion that DBS results in a random desychronization of brain rhythms, rather that DBS may regularize or 'tune' ongoing activity, presumably to different or higher frequencies than the pathological oscillations associated with disease. Speculatively, high-frequency DBS $(\geq 100 \mathrm{~Hz})$ may improve hyperkinetic involuntary movements such as tremor by dividing pathological central oscillations into more rapid, compact temporal units, such that they are not expressed in the periphery, based upon motor unit physiology and/or the biomechanical properties of the limbs.

Importantly, these EEG methods or their extensions might eventually represent a noninvasive biological marker for the physiological dose of DBS in the brain, regardless of whether the cortical rhythms associated with DBS are causally related to its therapeutic effect. Clearly, additional proof-of-concept research and prospective studies with appropriate masking would be required to validate such an approach. Such a biomarker could be applied to increase efficacy, decrease potential adverse effects and improve energy efficiency, not only in Parkinson's disease and essential tremor, but perhaps more substantially in diseases such as dystonia, obsessive-compulsive disorder, epilepsy and depression, where immediate proxies for clinical outcome such as tremor are often unavailable.

Financial \& competing interests disclosure
This work was supported by the US NIH (K23
NSO67053 to HC Walker). The authors have no other
relevant affiliations or financial involvement with any
organization or entity with a financial interest in or
financial conflict with the subject matter or materials
discussed in the manuscript apart from those
disclosed.
No writing assistance was utilized in the production
of this manuscript.

This work was supported by the US NIH (K23 NS067053 to HC Walker). The authors have no other relevant affiliations or financial involvement with any organization or entity with a financial interest in or financial conflict with the subject matter or materials discussed in the manuscript apart from those disclosed. of this manuscript.

\section{References}

1. Walker HC, Huang H, Gonzalez CL et al. Short latency activation of cortex during clinically effective subthalamic deep brain stimulation for Parkinson's disease. Mov. Disord. 27(7), 864-873 (2012).

2. Walker HC, Huang H, Gonzalez CL et al. Short latency activation of cortex by clinically effective thalamic brain stimulation for tremor. Mov. Disord. 27(11), 1404-1412 (2012).

3. Baker KB, Montgomery EB Jr, Rezai AR, Burgess R, Luders HO. Subthalamic nucleus deep brain stimulus evoked potentials: physiological and therapeutic implications. Mov. Disord. 17(5), 969-983 (2002).

4. MacKinnon CD, Webb RM, Silberstein P et al. Stimulation through electrodes implanted near the subthalamic nucleus activates projections to motor areas of cerebral cortex in patients with Parkinson's disease. Eur. J. Neurosci. 21(5), 1394-1402 (2005).
5. Tisch S, Rothwell JC, Zrinzo L, Bhatia KP, Hariz M, Limousin P. Cortical evoked potentials from pallidal stimulation in patients with primary generalized dystonia. Mov. Disord. 23(2), 265-273 (2008).

6. Kuriakose R, Saha U, Castillo G et al. The nature and time course of cortical activation following subthalamic stimulation in Parkinson's disease. Cereb. Cortex 20(8), 1926-1936 (2009).

7. Li S, Arbuthnott GW, Jutras MJ, Goldberg JA, Jaeger D. Resonant antidromic cortical circuit activation as a consequence of high-frequency subthalamic deep-brain stimulation. J. Neurophysiol. 98(6), 3525-3537 (2007).

8. Sirota MG, Swadlow HA, Beloozerova IN. Three channels of corticothalamic communication during locomotion. J. Neurosci. 25(25), 5915-5925 (2005).

9. Nambu A, Tokuno H, Takada M. Functional significance of the cortico-subthalamopallidal 'hyperdirect' pathway. Neurosci. Res. 43(2), 111-117 (2002).
10. Braak H, Del Tredici K. Cortico-basal ganglia-cortical circuitry in Parkinson's disease reconsidered. Exp. Neurol. 212(1), 226-229 (2008).

11. Gradinaru V, Mogri M, Thompson KR, Henderson JM, Deisseroth K. Optical deconstruction of parkinsonian neural circuitry. Science 324(5925), 354-359 (2009).

12. Lind G, Schechtmann G, Lind C, Winter J, Meyerson BA, Linderoth B. Subthalamic stimulation for essential tremor. Short- and long-term results and critical target area. Stereotact. Funct. Neurosurg. 86(4), 253-258 (2008).

13. Blomstedt P, Sandvik U, Tisch S. Deep brain stimulation in the posterior subthalamic area in the treatment of essential tremor. Mov. Disord. 25(10), 1350-1356 (2010).

14. Moro E, Schwalb JM, Piboolnurak P et al. Unilateral subdural motor cortex stimulation improves essential tremor but not Parkinson's disease. Brain 134(Pt 7), 2096-2105 (2011). 


\section{Editorial Walker \& Guthrie}

15. Boertien T, Zrinzo L, Kahan J et al. Functional imaging of subthalamic nucleus deep brain stimulation in Parkinson's disease. Mov. Disord. 26(10), 1835-1843 (2011).

16. Logothetis NK What we can do and what we cannot do with fMRI. Nature 453(7197), 869-878 (2008).

17. Wylie SA, Ridderinkhof KR, Elias WJ et al. Subthalamic nucleus stimulation influences expression and suppression of impulsive behaviour in Parkinson's disease. Brain 133(Pt 12), 3611-3624 (2010).

18. Montgomery EB Jr. Effects of GPi stimulation on human thalamic neuronal activity. Clin. Neurophysiol. 117(12), 2691-2702 (2006).

19. Kuncel AM, Cooper SE, Wolgamuth BR et al. Clinical response to varying the stimulus parameters in deep brain stimulation for essential tremor. Mov. Disord. 21(11), 1920-1928 (2006).
20. Carlson JD, Cleary DR, Cetas JS, Heinricher MM, Burchiel KJ. Deep brain stimulation (DBS) does not silence neurons in subthalamic nucleus in Parkinson's patients. J. Neurophysiol. 103(2), 962-927 (2009).

21. Walker HC, Watts RL, Schrandt CJ et al. Activation of subthalamic neurons by contralateral subthalamic deep brain stimulation in Parkinson disease. J. Neurophysiol. 105(3), 1112-1121 (2010). 